\title{
UEBER SYSTEME VON DIFFERENTIALGLEICHUNGEN DENEN VIERFACH PERIODISCHE FUNCTIONEN GENÜGE LEISTEN*
}

VON

\section{MARTIN KRAUSE}

In der Theorie der elliptischen Functionen haben sich die linearen Differentialgleichungen von Bedeutung gezeigt, deren Coefficienten gewöhnliche doppelt periodische Functionen sind, während die Integrale sich als doppelt periodische Functionen zweiter Art darstellen lassen. Insbesonders sind zwei Gleichungen in einer Reihe von Arbeiten untersucht worden, die Lamesche $\dagger$ und die PiCardsche $\ddagger$ Differentialgleichung zweiter Ordnung. Nachdem Verfasser $\S$ versucht hat, im Gebiete der Systeme von Differentialgleichungen zweiter Ordnung, deren Coefficienten vierfach periodische Functionen sind, diejenigen herauszugreifen, welche als einfachstes Analagon zu der LAMÉschen Differentialgleichung angesehen werden können, sollen im Folgenden Systeme von Differentialgleichungen aufgestellt werden, welche als Analogon zu der Picardschen Differentialgleichung zweiter Ordnung gelten können. Die Anschauungen, welche Verfasser zu seinen Resultaten geführt haben, beruhen auf den Arbeiten von Herrn Fuchs, insbesondere derjenigen, welche sich in den Nachrichten der Göttinger Gesellschaft der Wissenschaften vom Jahre 1878 und im 81sten Bande des Crelleschen Journals vorfinden.

\section{$\S 1$.}

Als Ausgangspunkt der Theorie wählen wir die Function :

$$
Z=G(z)^{\frac{1}{2}} e^{c \int_{G(z) \sqrt{ } R(z)}^{z^{\nu+1} d z}}
$$

wobei $G(z)$ eine ganze Function von $\approx$ aus lauter ungleichen Factoren bedeutet, welche die Form hat:

\footnotetext{
* Presented to the Society (Chicago) April 14, 1900. Received for publication April 5, 1900. †Siehe hierüber vor allem HeRmite : Sur quelques applications des fonctions elliptiques. Paris 1885.

$\ddagger$ PICARD: Sur quelques applications de la théorie des fonctions elliptiques, Comptes Rendus, Band 89.

\& Sur les systèmes d'équations différentielles auxquels satisfont les fonctions quadruplement periodiques de seconde espèce, Comptes Rendus, 1898.

Ueber verallg:meinerte Lamé-Hermitesche Differentialgleichungen für den Fall zweitr veränderlichen Grössen, Berichte der Leipziger Gesellschaft der Wissenschaften, 1898.
} 


$$
G(z)=\left(z-t_{1}\right)\left(z-t_{2}\right) \cdots\left(z-t_{m}\right)=\sum_{s=0}^{s=m} p_{s} z^{m-5},
$$

während unter $R(z)$ der Ausdruck verstanden wird :

$$
R(z)=z(1-z)\left(1-\kappa^{2} z\right)\left(1-\lambda^{2} z\right)\left(1-\mu^{2} z\right)=\sum_{s=0}^{s=5} r_{s} z^{5-s} .
$$

Diese Function $Z$ leistet dann der Differentialgleichung Genüge :

$$
R(z) \frac{d^{2} Z}{d z^{2}}+\left(\frac{1}{2} R^{\prime}(z)-\frac{(\nu+1) R(z)}{z}\right) \frac{d Z}{d z}=\underset{4 G}{Z} M
$$

$$
M=G^{\prime}(z) R^{\prime}(z)-\frac{2(\nu+1) G^{\prime}(z) R(z)}{z}+2 G^{\prime \prime}(z) R(z)+\frac{4 c^{2} z^{2 \nu+2}-R(z) G^{\prime}(z)^{2}}{G(z)} .
$$

Wir wollen nun die Bedingung hinzunehmen, dass der Coefficient von $Z$ vom Unendlichkeitspunkt abgesehen den Nullpunkt als einzigen singulären Punkt besitzen soll, dann muss jedenfalls die Grösse:

$$
G^{\prime}(z)^{2} R(z)-4 c^{2} z^{2 v+2}
$$

durch $G(z)$ theilbar sein, oder also die Form haben:

$$
z^{2 v+2} G(z) \phi(z),
$$

wobei dann $z^{2 v+2} \phi(z)$ eine ganze Function von $z$ bedeutet. Nehmen wir an, dass

$$
2 \nu+2<2 m+3
$$

ist, so wird der Grad von $z^{2 \nu+2} \phi(z)$ gleich $m+3$ sein, so zwar, dass gesetzt werden kann :

$$
\phi(z)=\frac{c_{-2 v-1}}{z^{2 v+1}}+\cdots+c_{m-2 v+1} z^{m-2 v+1} .
$$

Es ergiebt sich dann die Beziehung:

$$
\frac{G^{\prime}(z)^{2} R(z)}{z^{2 v+2}}-4 c^{2}=G(z) \phi(z) .
$$

Wir differentiiren links und rechts nach $\approx$. Der Differentialquotient auf der linken Seite nimmt die Form an :

$$
G^{\prime}(z) \phi_{1}(z)
$$

während er auf der rechten Seite wird :

$$
G(z) \phi^{\prime}(z)+G^{\prime}(z) \phi(z) \text {. }
$$

Da $G(z)$ und $G^{\prime}(z)$ keinen gemeinsamen Factor besitzen, so muss eine Gleichung von der folgenden Form bestehen: 


$$
z^{2 v+2} \phi^{\prime}(z)=G^{\prime}(z)\left(A z^{3}+B z^{2}+C z+D\right)
$$

oder also wir erhalten die Beziehung:

$$
z^{2 v+2} \phi_{1}(z)=G(z)\left(A z^{3}+B z^{2}+C z+D\right)+z^{2 v+2} \phi(z) .
$$

Andrerseits ergiebt sich, wie aus der Definition von $\phi_{1}(z)$ folgt, die Gleichung :

$$
\begin{aligned}
z^{2 \nu+2} \phi_{1}(z)=2 G^{\prime \prime}(z) R(z)-G^{\prime}(z)\{2 \nu & +1+2 \nu r_{3} z+(2 \nu-1) r_{2} z^{2} \\
& \left.+(2 \nu-2) r_{1} z^{3}+(2 \nu-3) r_{0} z^{4}\right\} .
\end{aligned}
$$

Aus dieser Gleichung folgt für $\phi_{1}(\approx)$ die Form :

$$
\phi_{1}(z)=\sum_{s=-3}^{s=m} q_{s} z^{m-2 \nu-s-2}
$$

wobei $q_{s}$ den Werth besitzt:

$$
q_{s}=\sum_{\epsilon=-1}^{\epsilon=3}(m-s-\epsilon)(2 m-2 \nu-2 s-\epsilon-2) p_{s+\epsilon} r_{3-\epsilon} .
$$

Differentiiren wir in Gleichung (5) beide Seiten nach $z$ und berücksichtigen Gleichung (4), so erhalten wir die Beziehung:

$$
\phi_{1}^{\prime}(z)=\sum q_{s}^{\prime} z^{m-2 v-s-3},
$$

wobei gesetzt ist :

$$
\begin{aligned}
q_{s}^{\prime}=(2 m- & 2 s-2 \nu-5) p_{s+3} A+(2 m-2 s-2 \nu-4) p_{s+2} B \\
+ & (2 m-2 s-2 \nu-3) p_{s+1} C+(2 m-2 s-2 \nu-2) p_{s} D .
\end{aligned}
$$

Aus Gleichung (6) ergiebt sich aber für $\phi_{1}^{\prime}(z)$ ein zweiter Ausdruck, nämlich :

$$
\phi_{1}^{\prime}(z)=\sum(m-2 \nu-s-2) q_{s} z^{m-2 \nu-s-3} .
$$

Die Vergleichung der beiden für $\phi_{1}^{\prime}(z)$ gefundenen Werthe führt dann zu der Recursionsformel :

$$
(m-2 \nu-s-2) q_{s}=q_{s}^{\prime} .
$$

Sondern wir die Werthe $m=\nu$ und $m=\nu+1$ ab und setzen für $s$ der Reihe nach : $-3,-2,-1,0$, so ergeben sich für die Grössen $A, B, C, D$ die Werthe:

$$
\begin{gathered}
A=m(m-2 \nu+1) r_{0}, \\
B=m(m-2 \nu) r_{1}-(2 m-2 \nu-1) p_{1} r_{0}, \\
C=m(m-2 \nu-1) r_{2}-2(m-\nu-1) p_{1}\left(r_{1}-p_{1} r_{0}\right)-2(2 m-2 \nu-3) p_{2} r_{0}, \\
D=m(m-2 \nu-2) r_{3}-(2 m-2 \nu-3) p_{1}\left(r_{2}-p_{1} r_{1}+p_{1}^{2} r_{0}\right) \\
-2(2 m-2 \nu-4) p_{2} r_{1}+(6 m-6 \nu-11) p_{1} p_{2} r_{0}-3(2 m-2 \nu-5) p_{3} r_{0} .
\end{gathered}
$$$$
D=m(m-2 \nu-2) r_{3}-(2 m-2 \nu-3) p_{1}\left(r_{2}-p_{1} r_{1}+p_{1}^{2} r_{0}\right)
$$ 
Aus den folgenden Gleichungen für $s=1,2, \ldots, m$ sind die Grössen $p$ mit Ausnahme einer einzigen bestimmt, welche willkührlich bleibt.

Die Differentialgleichung (2) nimmt die Form an :

$$
R(z) \frac{d^{2} Z}{d z^{2}}+\left(\frac{1}{2} R^{\prime}(z)-\frac{(\nu+1) R(z)}{z}\right) \frac{d Z}{d z}=\frac{Z}{4}\left(A z^{3}+B z^{2}+C z+D\right)
$$

Die beiden Integrale besitzen die Form :

$$
G(z)^{\frac{1}{2}} e^{ \pm c \int \frac{z^{\nu+1} d z}{G(z) \sqrt{R(z)}}}
$$

wobei das positive Zeichen zu dem einen, das negative zu dem anderen Integral gehört.

$$
\S 2 .
$$

Wir haben bei den Erörterungen des vorigen Paragraphen die Ungleichheit gebraucht :

$$
2 m+3>2 \nu+2 \text {. }
$$

Im Uebrigen kann $m$ eine jede ganze positive Zahl bedeuten. Die Resultate vereinfachen sich etwas, wenn $m$ so bestimmt wird, dass

wird. Es geschieht das für

$$
A=0
$$

$$
m=2 \nu-1
$$

Mit diesem Werth von $m$ wollen wir weiter rechnen, bemerken aber, dass auch der allgemeine Fall im Prinzip genau so behandelt werden kann, wie der soeben angeführte specielle.

Wir wollen uns dann die Gleichung (13) der vorigen Paragraphen für zwei unabhängige Veränderliche gebildet denken, so dass wir die Gleichungen erhalten;

$$
\begin{aligned}
& R\left(z_{1}\right) \frac{d^{2} Z}{d z_{1}^{2}}+\left({ }_{1: 2}^{1} R^{\prime}\left(z_{1}\right)-\frac{(\nu+1) R\left(z_{1}\right)}{z_{1}}\right) \frac{d Z}{d z_{1}}=\frac{Z}{4}\left(B z_{1}^{2}+C z_{1}+D\right), \\
& R\left(z_{2}\right) \frac{d^{2} Z}{d z_{2}^{2}}+\left({ }_{2}^{1} R^{\prime}\left(z_{2}\right)-\frac{(\nu+1) R\left(z_{2}\right)}{z_{2}}\right) \frac{d Z}{d z_{2}}=\frac{Z}{4}\left(B z_{2}^{2}+C z_{2}+D\right) .
\end{aligned}
$$

Die Integrale der ersten haben die Form:

$$
Z_{1}^{(1)}=G\left(z_{1}\right)^{\frac{1}{2}} e^{c \int \frac{z_{1}^{\nu+1} d z_{1}}{G\left(z_{1}\right) \sqrt{\overline{R\left(z_{1}\right)}}}}, \quad Z_{1}^{(2)}=G\left(z_{1}\right)^{\frac{1}{2}} e^{-c \int \frac{z_{1} \nu+1}{G\left(z_{1}\right) \sqrt{R\left(z_{1}\right)}}}:
$$

die Integrale der zweiten können wir schreiben :

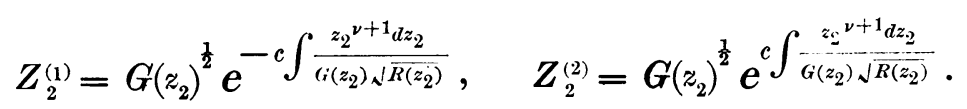


Die Functionen :

$$
Z^{(1)}=Z_{1}^{(1)} Z_{2}^{(1)} \text { und } Z^{(2)}=Z_{1}^{(2)} Z_{2}^{(2)}
$$

genügen dann jedenfalls beiden Differentialgleichungen.

Wir führen jetzt die hyperelliptischen Functionen nach RosenhaIN ein, indem wir setzen :

$$
\begin{aligned}
& 2 d u_{1}=\frac{d z_{1}}{\sqrt{R\left(z_{1}\right)}}-\frac{d z_{2}}{\sqrt{ } R\left(z_{2}\right)}, \\
& 2 d u_{2}=-\frac{z_{1} d z_{1}}{\sqrt{ } R\left(z_{1}\right)}+\frac{z_{2} d z_{2}}{\sqrt{ } R\left(z_{2}\right)} .
\end{aligned}
$$

Die Grössen $Z^{(1)}$ und $Z^{(2)}$ können dann auch als Functionen von $u_{1}$ und $u_{2}$ angesehen werden. Um das anzudeuten, wollen wir an Stelle des Buchstabens $Z$ uns den Buchstaben $\phi$ eingeführt denken. Die Differentialgleichungen (1) nehmen dann die Form an :

$$
\begin{aligned}
& \frac{\partial^{2} \phi}{\partial u_{1}^{2}}-2 z_{1} \frac{\partial^{2} \phi}{\partial u_{1} \partial u_{2}}+z_{1}^{2} \frac{\partial^{2} \phi}{\partial u_{2}^{2}}-\frac{2(\nu+1)}{z_{1}} \sqrt{R\left(z_{1}\right)} \frac{\partial \phi}{\partial u_{1}}+2 \nu \sqrt{R\left(z_{1}\right)} \frac{\partial \phi}{\partial u_{2}} \\
& =\left(B z_{1}^{2}+C z_{1}+D\right) \phi,
\end{aligned}
$$

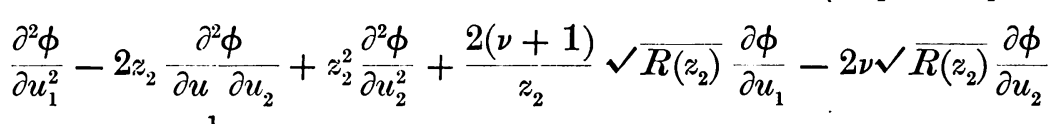

$$
\begin{aligned}
& =\left(B z_{2}^{2}+C z_{2}+D\right) \phi \text {. }
\end{aligned}
$$

Aus ihnen ergeben sich durch einfache Operationen die beiden Differentialgleichungen :

$$
\begin{aligned}
& \frac{\partial^{2} \phi}{\partial u_{1}^{2}}-s_{2} \frac{\partial^{2} \phi}{\partial u_{2}^{2}}-\frac{(\nu+1)}{s_{2} \kappa \lambda \mu} \frac{\partial}{\partial u_{1}} \frac{\vartheta_{1}^{2}(v)}{\vartheta_{0}^{2}(v)} \frac{\partial \phi}{\partial u_{1}}+\frac{\nu}{\kappa \lambda \mu} \frac{\partial}{\partial u_{2}} \frac{\vartheta_{1}^{2}(v)}{\vartheta_{0}^{2}(v)} \frac{\partial \phi}{\partial u_{2}} \\
& =\phi\left(-B s_{2}+D\right) \text {, }
\end{aligned}
$$

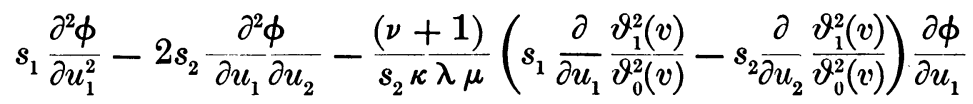

$$
\begin{aligned}
& +\frac{\nu}{\kappa \lambda \mu} \frac{\partial}{\partial u_{1}} \frac{\vartheta_{1}^{2}(v)}{\vartheta_{0}^{2}(v)} \frac{\partial \phi}{\partial u_{2}}=\phi\left(C s_{2}+D s_{1}\right) .
\end{aligned}
$$

In diesen Gleichungen ist gesetzt :

$$
\begin{gathered}
\left.s_{1}=\frac{1}{\kappa^{2}}+\frac{1}{\kappa^{2} \lambda^{2}} \frac{\mu^{2}}{\kappa^{4}} \frac{\theta_{1}^{\prime}\left(u_{1}\right)_{0}^{2}}{\vartheta_{0}^{2}} \frac{\vartheta_{1}^{2}(v)}{\vartheta_{0}^{2}(v)}+\frac{\theta_{3}^{\prime}\left(u_{2}\right)_{0}^{2}}{\vartheta_{0}^{2}} \frac{\vartheta_{3}^{2}(v)}{\vartheta_{0}^{2}(v)}\right), s_{2}=\frac{1}{\kappa \lambda \mu} \frac{\vartheta_{1}^{2}(v)}{\vartheta_{0}^{2}(v)}, \\
\theta_{a}^{\prime}\left(u_{\epsilon}\right)_{0}=\frac{\partial}{\partial u_{\epsilon}} \vartheta_{a}\left(v_{1}, v_{2}\right)_{\left(v_{1}, v_{2}\right)=(0,0)},
\end{gathered}
$$

während $v_{1}$ und $v_{2}$ die Argumente der Thetafunctionen bedeuten, welche den Argumenten $u_{1}$ und $u_{2}$ der hyperelliptischen Functionen entsprechen und mit ihnen durch die bekannten linearen Relationen verbunden sind. 
Es sind das zwei Differentialgleichungen mit vierfach periodischen Coefficienten, welche wir als Analogon zu der schon citirten Picardschen Differentialgleichung zweiter Ordnung im Gebiete der Ihetafunctionen zweier Veränderlichen ansehen können und zwar für den ersten Fall von $m$.

Die Darstellung zweier Integrale dieser Gleichungen durch Thetafunctionen kann ähnlich durchgeführt werden, wie es in den citirten Arbeiten des Verfassers für die verallgemeinerten LAMÉ-Hermiteschen Differentialgleichungen geschehen ist. Entwickeln wir :

$$
\frac{z_{1}^{v+1}}{G\left(z_{1}\right)} \text { und } \frac{z_{2}^{\nu+1}}{G\left(z_{2}\right)}
$$

in Partialbrüche und berücksichtigen die angegebenen Werthe von $c$, so gelangen wir zu Integralen derselben Form, wie sie an den angegebenen Orten durch Thetafunctionen dargestellt worden sind.

Wir müssen dazu den einzelnen Wurzeln der Gleichung :

$$
G(z)=0,
$$

d. h. den Grössen $t_{s}$ Parameter $a_{1}^{(s)}, a_{2}^{(*)}$ zuordnen, zwischen denen einerseits die Gleichung besteht:

$$
\vartheta_{3}\left(a_{1}^{(*)}, a_{2}^{(*)}\right)=0
$$

während sie andrerseits den Gleichungen Genüge leisten :

$$
\frac{\vartheta_{1}^{2}\left(a_{1}^{(s)}, a_{2}^{(s)}\right)}{\vartheta_{0}^{2}\left(a_{1}^{(s)}, a_{2}^{(s)}\right)}=\frac{\lambda \mu}{\kappa} t_{s}, \ldots .
$$

Unter solchen Umständen können wir die folgenden Sätze aussprechen :

I. Die Differentialgleichungen (3) ändern sich nicht, wenn an Stelle von $u_{1}, u_{2}$ resp. gesetzt wird: $-u_{1},-u_{2}$.

II. Die Coefficienten ihrer linken Seiten sind unabhängig von den Grössen $a_{1}^{(s)}, a_{2}^{(s)}$.

III. Zwei ihrer Integrale häben die F'orn:

$$
\begin{aligned}
\phi^{(1)} & =\prod_{s=1}^{s=m} \frac{\vartheta_{1}\left(v_{1}+a_{1}^{(s)}, v_{2}+a_{2}^{(s)}\right)}{\vartheta_{0}\left(v_{1}, v_{2}\right)} e^{\lambda_{s}}, \\
\phi^{(2)} & =\prod_{s=1}^{s=m} \frac{\vartheta_{1}\left(v_{1}-a_{1}^{(s)}, v_{2}-a_{2}^{(s)}\right)}{\vartheta_{0}\left(v_{1}, v_{2}\right)} e^{-\lambda_{s}},
\end{aligned}
$$

wobei unter Fortlassung des Index s gesetzt ist:

$$
\lambda=-\sum_{\epsilon=1}^{\epsilon=2} \frac{\partial}{\partial a_{\epsilon}} \log \vartheta_{1}\left(a_{1}, a_{2}\right) u_{\epsilon}-\underset{2 \lambda \mu}{\kappa} \partial \frac{\partial \vartheta_{1}^{2}\left(a_{1}, a_{2}\right)}{\partial a_{v}^{2}\left(a_{1}, a_{2}\right)} u_{1} .
$$

Auch die noch fehlenden beiden Integrale können durch Thetafunctionen dargestellt werden-es müssen dazu aber andere Argumente, als die bisherigen eingeführt werden. 